\title{
Multiple Representations of Perceptual Features for Texture Classification and Retrieval
}

\author{
G. Tamilpavai \\ Assistant Professor \\ Department of Computer Science and Engineering \\ Government College of Engineering, \\ Tirunelveli,India
}

\author{
S. Tamil Selvi \\ PhD. Professor \\ Department of Electronics and Communication \\ Engineering \\ National Engineering College, Kovilpatti, India
}

\begin{abstract}
Texture is a very important feature extremely used in various image processing problems. Human beings are used some texture based perceptual features to distinguish between textured images or regions. These Perceptual features are highly desirable for two reasons; they will be optimum in terms of feature selection and will be applicable to all kinds of textures. Some of the important perceptual features are coarseness, contrast, directionality and busyness. This paper proposed a new perception-based approach to content-based image classification and retrieval. The proposal is based on multiple representations: Original Image Representation and Autocorrelation Function Representation. The computational measures for textural features are computed both on original image and autocorrelated image. In order to validate these features measures, applied them for texture classification and retrieval on brodatz images. For texture classification, features computed on Multiple representation correctly classified the best matching class among the existing class in comparison with original representation based features and autocorrelation representation based features. K-Nearest Neighborhood classifier is used for this classification task. For texture retrieval, Multiple representation based features retrieved more number of relevant images in comparison with features derived from autocorrelation representation. Gower co-efficient of similarity is used to find the feature similarity between images in retrieval task. Thus this work attained good classification rate of $93.5 \%$ and better retrieval rate by using these estimated features on our approach.
\end{abstract}

\section{Keywords}

Multiple representations, perceptual features, texture, texture classification, texture retrieval.

\section{INTRODUCTION}

Texture refers to the spatial distribution of grey-levels and can be defined as the deterministic or random repetition of one or several primitives in an image. Texture feature are widely used for extraction of specific image properties such as coarseness and presence of edges.

A number of texture analysis methods have been proposed [1]-[15]. Haralick [16] categorized texture analysis methods into statistical methods, structural methods, and hybrid methods [17]-[21]. The drawbacks of almost all of these approaches are that they do not have general applicability and computational cost involved, either in terms of memory requirement, computation time or implementational complexity. In comparison, human visual perception seems to work perfectly for almost all types of textures. The reason for this mismatch between computational methods and human vision is that the majority of the computational methods use mathematical features that have no perceptual meaning.

Among the various works that have been done in the field of texture analysis [22], this paper is interested in dealing with human visual perception. In the literature, some works related to perceptual texture analysis have been done [23]-[27]. Tamura et al. [28] and Amadasum et al. [29] proposed, each, computational measures for some textural features such as coarseness, contrast, direction, busyness, regularity and complexity. They studied, then, the correspondence between the classification obtained with these computational measures and classification made by human subjects. The work of Tamura et al. [28] was based on the co-occurrence grey-level matrix (CGLM). The work of Amadasun et al. [29] was based on the neighborhood gray-tone difference matrix (NGTDM). The results they obtained with the computational measures were relatively good with respect to human classification. Ravishankar et al. [30] proposed a texture naming system, i.e. they try to determine the relevant dimensions of texture such as the three dimensional representations of color (RGB, HIS, etc).

The objective of this paper is to evaluate the computational measures for perceptual textural features. First, proposed a new method to estimate a set of perceptual textural features. Second, application of this proposed perceptual model to texture classification and retrieval task. Our approach is to combine the features which are computed on both original images and the autocorrelation function and applied them for classification and retrieval task to obtain very good classification and retrieval rate. It should be noted that the study reported here was tested on a sample of 8 images (Figure.1) from Brodatz database [31].

The rest of this paper is organized as follows. In section 2, we present definitions of the set of perceptual textural features we are considering in this study; in section 3, the autocorrelation function on which our work is based is presented; in section 4, computational measures corresponding to the perceptual textural features are presented; in section 5, application of perceptual features to texture classification is presented with implementation result; in section 6 , application of perceptual features to texture retrieval is presented with experimental result; in section 7 and 8 , a conclusion and future work are given respectively. 


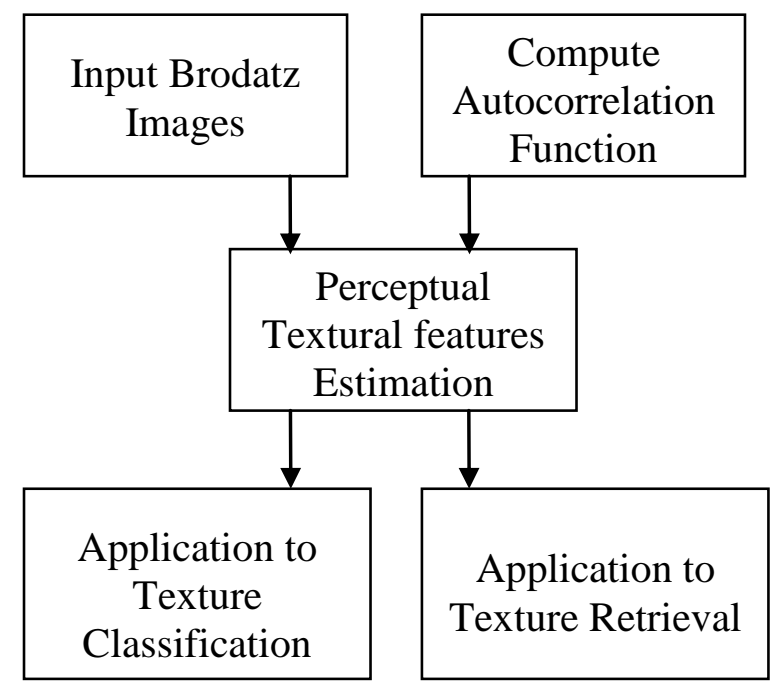

Figure 1: Block diagram of proposed system

Figure 1 shows the block diagram of this work. Autocorrelation function is represented on each input Brodatz image. Computational measures for perceptual textural features are computed both on original image and autocorrelation representation. The feature measures obtained from these two representations are combined and applied for texture classification and retrieval task.

\section{PERCEPTUAl TeXTURAL FEATURES}

In our work, we have considered four perceptual features. In the following, we give conceptual definitions of each of these features.

Coarseness is the most important feature that determines the existence of texture in an image. Coarseness measures the size of the primitives that constitute the texture[32][33]. A coarse texture is composed of large primitives and characterized by a high degree of local uniformity of grey-levels. A fine texture is constituted by small primitives and is characterized by a high degree of local variations of grey-levels.

Directionality is a global property in an image. Directionality measures the degree of visible dominant orientation in an image[34]. An image can have one or several dominant orientation(s) or no orientation at all. In the latter case, it is said isotropic. The orientation is influenced by the shape of primitives as well as by their placement rules.

Contrast measures the degree of clarity with which one can distinguish between different primitives in a texture[35]. A well-contrasted image is an image in which primitives are clearly visible and separable. The contrast is influenced by the grey-levels in the image, the ratio of white and black in the image and the intensity change frequency of grey-levels.

Busyness refers to the intensity changes from a pixel to its neighborhood; a busy texture is a texture in which the intensity changes are slow and gradual. Therefore busyness is related to spatial frequency of the intensity changes in an image. If intensity changes are very small, they risk to be invisible. Consequently, the amplitude of the intensity changes has also an influence on busyness. Busyness has a reverse relationship with coarseness.

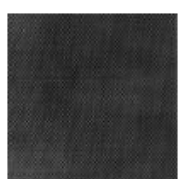

D6

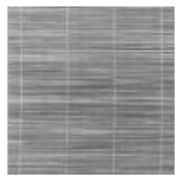

D49

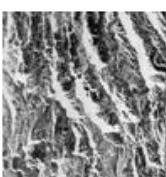

D13

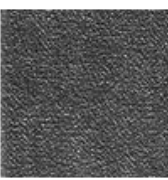

D57

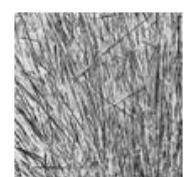

D15

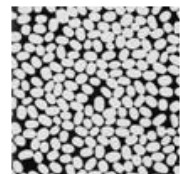

D75

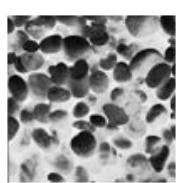

D31

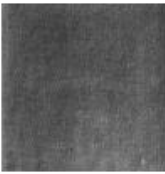

D77
Figure 2: Brodatz images used in this work

\section{AUTOCORRELATION FUNCTION REPRESENTATION}

The autocorrelation function of an image can be used to assess the amount of regularity as well as fineness of the texture present in the image, denoted as $f\left(\delta_{\mathrm{i}}, \delta_{\mathrm{j}}\right)$. For an $\mathrm{n} \times \mathrm{m}$ image I is defined as follows[18]:

$$
f\left(\delta_{i}, \delta j\right)=\frac{1}{\left(n-\delta_{i}\right)\left(m-\delta_{j}\right)} \sum_{i=1}^{n-\delta_{i}} \sum_{j=0}^{m-\delta_{j}} I(i, j) I\left(i+\delta_{i}, j+\delta_{j}\right)
$$

where $1 \leq \delta_{\mathrm{i}} \leq \mathrm{n}$ and $1 \leq \delta_{\mathrm{j}} \leq \mathrm{m}$. $\delta_{\mathrm{i}}$ and $\delta_{\mathrm{j}}$ represent shift on rows and columns, respectively.

This function is related to the size of the texture primitive. If the texture is coarse, then the autocorrelation function will drop of slowly; otherwise, it will drop off very rapidly. For regular textures, the autocorrelation function will exhibit peaks and valleys.

\section{COMPUTATIONAL MEASURES FOR PERCEPTUAL FEATURES}

The general estimation process of computational measures simulating human visual perception is as follows[36]-[38]

1) The autocorrelation $f(i, j)$ is computed on image $I(i, j)$.

2) Then, the convolution of the autocorrelation function and the gradient of the Gaussian function are computed in a separable way (according to rows and columns). Two functions are then obtained.

3) Based on these functions, computational measures for each perceptual features are computed as described in the following subsections.

For multiple representations, the computational measures presented in the following are computed on both the original images and the autocorrelated images.

\subsection{Coarseness Estimation}

When considering the autocorrelation function, we can notice that coarseness is saved in the corresponding autocorrelation function. Therefore, number of extrema in the autocorrelation function determines coarseness of a texture.

Coarseness, denoted $\mathrm{C}_{\mathrm{s}}$, is estimated as the average number of maxima in the autocorrelated images and original images. A coarse texture will have a small number of maxima and a fine texture will have a large number of maxima. Coarseness $\mathrm{C}_{\mathrm{s}}$ can be written as 


$$
\mathrm{C}_{\mathrm{s}}=\frac{1}{0.5 \times\left(\frac{\sum_{\mathrm{i}=1}^{\mathrm{n}} \sum_{\mathrm{j}=1}^{\mathrm{m}} \operatorname{Max}_{\mathrm{x}}(\mathrm{i}, \mathrm{j})}{\mathrm{n}}+\frac{\sum_{\mathrm{j}=1}^{\mathrm{m}} \sum_{\mathrm{i}=1}^{\mathrm{n}} \operatorname{Max}_{\mathrm{y}}(\mathrm{i}, \mathrm{j})}{\mathrm{m}}\right)}
$$

\subsection{Contrast Estimation}

If the image is well-contrasted, the value of autocorrelation function decreases quickly; otherwise, it decreases slowly. Therefore, amplitude $\mathrm{M}$ of the gradient of the autocorrelation function determines contrast.

Contrast, denoted $\mathrm{C}_{\mathrm{t}}$, is estimated as the product of the average module $\mathrm{M}_{\mathrm{a}}$ of the gradient of the autocorrelation function, the percentage of points $\frac{\mathrm{N}_{\mathrm{t}}}{\mathrm{nxm}}$ having a module greater than a threshold $t$ and the coarseness $C_{s}$. Coarseness is introduced here to reflect the fact that a coarse texture is more clearly visible than a fine texture. So contrast is given by

$$
\mathrm{C}_{\mathrm{t}}=\frac{\mathrm{M}_{\mathrm{a}} \times \mathrm{N}_{\mathrm{t}} \times \mathrm{C}_{\mathrm{s}}^{\frac{1}{\alpha}}}{\mathrm{n} \times \mathrm{m}}
$$

$\frac{1}{\alpha}$ is a parameter used to make $\mathrm{C}_{\mathrm{s}}$ having sense against the quantity $\frac{M_{\mathrm{a}} \mathrm{x} N_{\mathrm{t}}}{n \times m}$.

\subsection{Direction Estimation}

Two parameters are estimated here: the orientation $\theta$ and the degree of directionality. The Orientation $\theta$ is estimated as the orientation of the gradient of the autocorrelated image or original image. It is given by

$$
\theta=\arctan \mathrm{G}_{\mathrm{y}} / \mathrm{G}_{\mathrm{x}}
$$

The degree of directionality $N_{\theta_{d}}$ is estimated as the number of points having the dominant orientation $\theta_{\mathrm{d}}$. The dominant orientation $\theta_{\mathrm{d}}$ is the orientation of the largest number of pixels in an image having a module greater than a threshold $t$. $N_{\theta_{d}}$ can be expressed as follow

$$
\mathrm{N}_{\theta_{\mathrm{d}}}=\frac{\sum_{\mathrm{i}=1}^{\mathrm{n}} \sum_{\mathrm{j}=1}^{\mathrm{m}} \theta_{\mathrm{d}}(\mathrm{i}, \mathrm{j})}{\mathrm{n} \times \mathrm{m}}
$$

\subsection{Busyness Estimation}

Busyness is related to coarseness in the reverse order, that is when the coarseness is high, the busyness is low. So busyness is estimated by using coarseness as follow

$$
\mathrm{B}_{\mathrm{s}}=1-\mathrm{C}_{\mathrm{s}}^{\frac{1}{\alpha}}
$$

$\frac{1}{\alpha}$ is a parameter used to make $C_{s}$ having sense against the quantity 1 .

\section{APPLICATION TO TEXTURE CLASSIFICATION}

This experiment was carried out to assess the performance of the perceptual features in texture classification task. Computational features presented in this paper are applied in classification task on Brodatz database. The samples used are D13, D57, D77, D6, D75, D49, D31, and D15. Three kinds of classifications are performed in this work namely original representation based classification, autocorrelation representation based classification and multiple representation based classification. Actual classification task is achieved by K-Nearest Neighborhood classifier.

\subsection{K-Nearest Neighborhood classifier}

This classifier is used to identify the best matching class among the existing class by using nearest neighbor method. The syntax of knnclassify is defined as follows

$$
\text { Class }=\text { knnclassify }(\text { Sample, Training, Group) }
$$

Where,

Sample is the matrix whose values represent the set of features which are obtained during testing phase. Sample must have same number of columns as Training.

Training is the matrix whose values represent the set of features which are obtained during training phase.

Group is the vector whose distinct values define different class

\subsection{Implementation}

Texture classification consist of two phases namely training phase and testing phase. The database contain sample of 8 brodatz images. In training phase, each of these samples is divided into 9 nonoverlapping tiles to obtain 72 subimages of size $128 \times 128$ ( 8 images $\times 9$ titles per image). During this phase, leaving out four subimages for each category at a time and training the classifier on the remaining five. Thus, training set and testing set contains 40 ( 8 images $x 5$ titles per image) and 32 (8 images x 4 titles per image) subimages respectively. In testing phase, four untrained subimages for each class in the testing set are presented to the classifier to identify the best matching class.

\subsection{Classification Based on 9 Subimages from Each class}

\subsubsection{Training phase for original representation}

Each database image is divided into 9 subimages. Five subimages from each category are stored in training set and remaining goes for testing purpose. Computational measures for perceptual features are computed on training set images. Thus we are obtained the feature set based on original representation.

\subsubsection{Training phase for autocorrelation representation}

Each database image is divided into 9 subimages. Five subimages from each category are stored in training set and remaining. Autocorrelation function is computed on each image in the training set and perceptual features are estimated. Thus we are obtained the feature set based on autocorrelation representation.

\subsubsection{Testing phase for original representation}

Perceptual features are estimated on each image in the testing set and obtained the feature set. Knnclassifier is used to find the best matching samples for the testing set images among the existing class in the training set. This is achieved by passing the feature sets which are obtained during training and testing phase for original representation.

\subsubsection{Testing phase for autocorrelation representation}

Autocorrelation function is represented on each image in the testing set and features are estimated at a time. Thus obtained the feature set. Knnclassifier is used to find the best matching samples for the testing set images among the existing class in the training set. This is achieved by passing the feature sets which are obtained during training and testing phase for autocorrelation representation. 


\subsubsection{Testing phase for multiple representation} Perceptual features are estimated on each image in the testing set and obtained the feature set on original images. Then, Autocorrelation function is represented on testing set images and features are estimated at a time. Thus obtained the feature set on autocorrelation representation. Knnclassifier is used to find the best matching samples for the testing set images among the existing class in the training set. This is achieved by passing the feature sets which are obtained during training phase for original and autocorrelation representation and testing phase for multiple representations.

Classification results are shown in table I. It clearly shows that knnclassifier obtained very good accurate rate in testing phase for multiple representation in comparison with other testing phases. Accuracy rate is defined as follows

Accuracy rate $=$

Total number of correct matching class for testing class Total number of subimages on testing set

Overall classification performances are shown in figure 3 . On these three types of classification, multiple representation based classification shows better accuracy rate in comparison with other two types of classification. It clearly shows that we have obtained more number of correctly classified samples for each category by our approach.

\subsection{Implementation Result}

Table I: Classification Results

\begin{tabular}{|c|c|}
\hline $\begin{array}{c}\text { Classification Based } \\
\text { on }\end{array}$ & Accuracy Rate \\
\hline $\begin{array}{c}\text { Original } \\
\text { Representation }\end{array}$ & 84.375 \\
\hline $\begin{array}{c}\text { Autocorrelation } \\
\text { Representation }\end{array}$ & 81.25 \\
\hline $\begin{array}{c}\text { Multiple } \\
\text { Representation }\end{array}$ & 93.75 \\
\hline
\end{tabular}

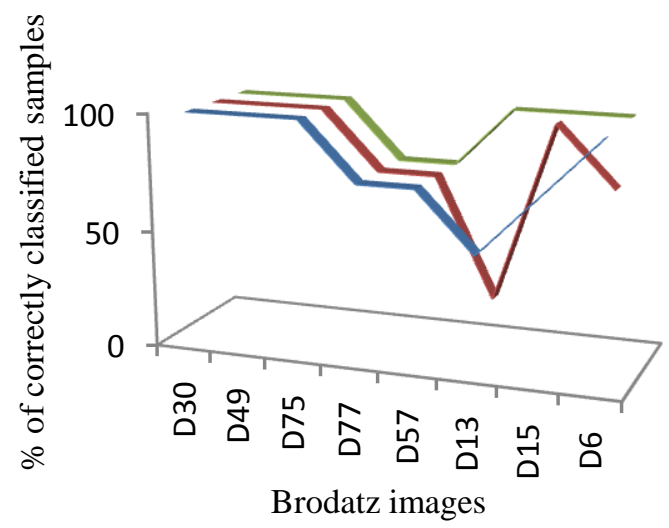

- Original representation based classification

- Autocorrelation representation based classification

Multiple representation based classification

\section{APPLICATION TO TEXTURE RETRIEVAL}

This experiment was carried out to assess the performance of the features in an actual retrieval task. Computational features presented in this paper are applied in texture retrieval on Brodatz database. The samples used are D13, D57, D77, D6, D75, D49, D31, and D15. Two kinds of retrieval are performed in this work. Retrieval performances are based on the number of subimages derived from each image in the database. Similarity measure used in this work is Gower coefficient of similarity.

\subsection{Gower Coefficient of Similarity}

The similarity between two texture images $\mathrm{GS}_{\mathrm{ij}}$, which are represented by $i$ and $j$ based on all features can be defined as

$$
\mathrm{GS}_{\mathrm{ij}}=\frac{\sum_{\mathrm{k}=1}^{4} \mathrm{~S}_{\mathrm{ij}}{ }^{(\mathrm{K})}}{4}
$$

Where, $\mathrm{s}_{\mathrm{ij}}{ }^{(\mathrm{k})}$ represent the similarity between two images on specific feature $\mathrm{k}$.

Quantity $\mathrm{S}_{\mathrm{ij}}^{(\mathrm{k})}$ is defined as follows

$$
S_{i j}^{(k)}=1-\frac{\left|x_{i k}-x_{j k}\right|}{R_{k}}
$$

Where, $x_{i k}$ is the feature $k$ on given input image

$\mathrm{x}_{\mathrm{jk}}$ is the feature $\mathrm{k}$ on $\mathrm{jth}$ image in the database

$\mathrm{R}_{\mathrm{k}}$ is the difference between maximum and minimum value of feature $\mathrm{k}$ on feature set $\mathrm{X}$.

Quantity $\mathrm{R}_{\mathrm{k}}$ is defined as follows

$$
\mathrm{R}_{\mathrm{k}}=\operatorname{Max}\left(\mathrm{X}_{\mathrm{k}}\right)-\operatorname{Min}\left(\mathrm{X}_{\mathrm{k}}\right)
$$

\subsection{Implementation}

Retrieval task consists of training and testing phase. In training phase, perceptual features are estimated from original image representation and autocorrelation function representation. In testing phase, autocorrelation representation based retrieval and multiple representation based retrieval are carried out.

\subsection{Retrieval Based on 9/25 Subimages from Each class}

6.3.1 Training phase for original representation In the database, each sample is divided into $9 / 25$ subimages of size $128 \times 128$. Computational measures of perceptual features are estimated from these subimages. Thus obtained the feature set based on original representation.

\subsubsection{Training phase for autocorrelation representation} In the database, each sample is divided into 9/25 subimages of size $128 \times 128$. Autocorrelation function is computed on subimages. Computational measures of perceptual features are estimated from these autocorrelated subimages. Thus obtained the feature set based on autocorrelation representation.

Figure 3: Performance measure of texture classification 


\subsubsection{Testing phase for autocorrelation representation}

Autocorrelation function is represented on the image which is given as input. Four features are estimated from this autocorrelated image. Gower coefficient of similarity is measured the similarity between these four features to the feature set which is obtained during the training phase for autocorrelation representation.

\subsubsection{Testing phase for multiple representation}

Four perceptual features are estimated from the image which is given as input. On that image, autocorrelation function is represented and four features are computed. Thus eight features are obtained. Similarity measurement is defined between these eight features to the feature sets which are obtained during the training phase for original representation and autocorrelation representation.

Retrieval results are shown in tables 1 and table 2. Table 1 shows the retrieval result for 9 subimages from each class. If there $\mathrm{n}$ images in the database, then the total number of retrievd images is $9 *$ n. Table 2 shows the retrieval result for 25 subimages from each class. If the total number of images in the database is $\mathrm{n}$, then the total number of retrieved images is $25 * \mathrm{n}$. Retrieval rate is defines as follow,

$$
\text { Retrieval Rate }=\frac{\text { No.of Relevant images retrieved }}{\text { Total number of retrieved images }}
$$

Overall retrieval performances are shown in figure 4 and figure 5. In both kind of retrievals, multiple representation based retrieval shows better retrieval rate than autocorrelation based retrieval. It clearly shows that we have retrieved more number of relevant images for our approach.

\subsection{Implementation result}

\begin{tabular}{|c|c|c|c|}
\hline \multirow{2}{*}{$\begin{array}{c}\text { Total No. } \\
\text { of Images } \\
\text { in Database }\end{array}$} & $\begin{array}{c}\text { Total No. of } \\
\text { Retrieved } \\
\text { Images }\end{array}$ & \multicolumn{2}{|c|}{$\begin{array}{c}\text { No. of Relevant Images } \\
\text { Retrieved Based on }\end{array}$} \\
\cline { 3 - 4 } & 162 & A & B \\
\hline 27 & 243 & 161 & 154 \\
\hline 36 & 324 & 222 & 275 \\
\hline 45 & 405 & 284 & 343 \\
\hline 54 & 486 & 341 & 410 \\
\hline 63 & 567 & 422 & 491 \\
\hline 72 & 648 & 478 & 556 \\
\hline
\end{tabular}

A) Autocorrelation representation

B) Multiple representation

Table II: Retrieval result based on 9 subimages from each class

\begin{tabular}{|c|c|c|c|}
\hline \multirow{2}{*}{$\begin{array}{c}\text { Total No. } \\
\text { of Images } \\
\text { in Database }\end{array}$} & \multirow{2}{*}{$\begin{array}{c}\text { Total No. of } \\
\text { Retrieved } \\
\text { Images }\end{array}$} & \multicolumn{2}{|c|}{$\begin{array}{c}\text { No. of Relevant Images } \\
\text { Retrieved Based on }\end{array}$} \\
\cline { 3 - 4 } & 1250 & A & B \\
\hline 50 & 1875 & 1244 & 1562 \\
\hline 75 & 2500 & 1790 & 2146 \\
\hline 100 & 3125 & 2176 & 2635 \\
\hline 125 & 3750 & 2801 & 3260 \\
\hline 150 & 4375 & 3274 & 3789 \\
\hline 175 & 5000 & 3806 & 4407 \\
\hline 200 & & & \\
\hline
\end{tabular}

A) Autocorrelation representation

B) Multiple representation

Table III: Retrieval result based on 25 subimages from each class

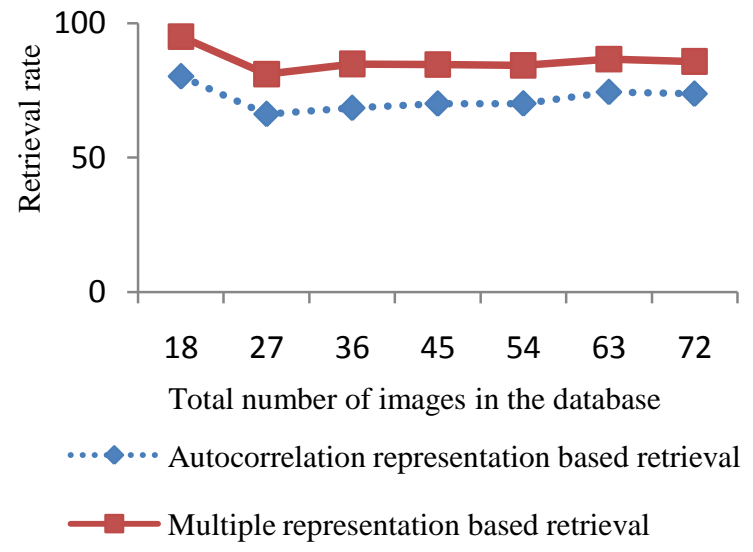

Figure 4: Performance measure of retrieval based on 9 subimages from each class

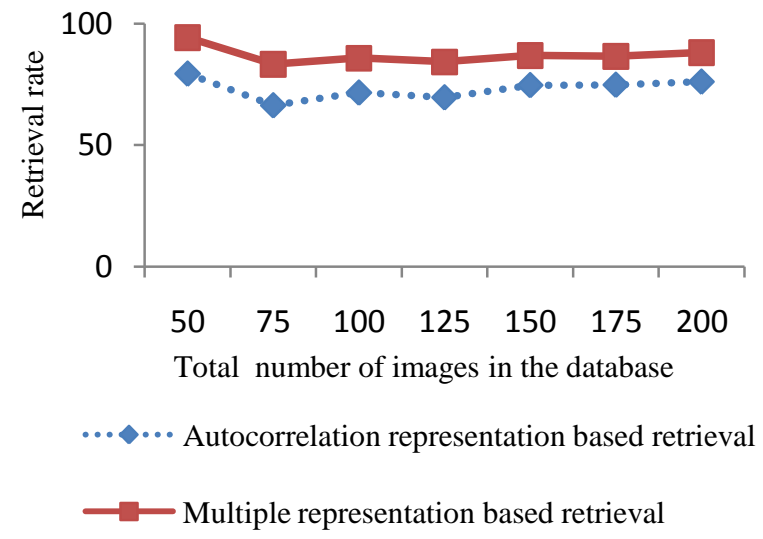

Figure 5: Performance measure of retrieval based on 25 subimages from each class 


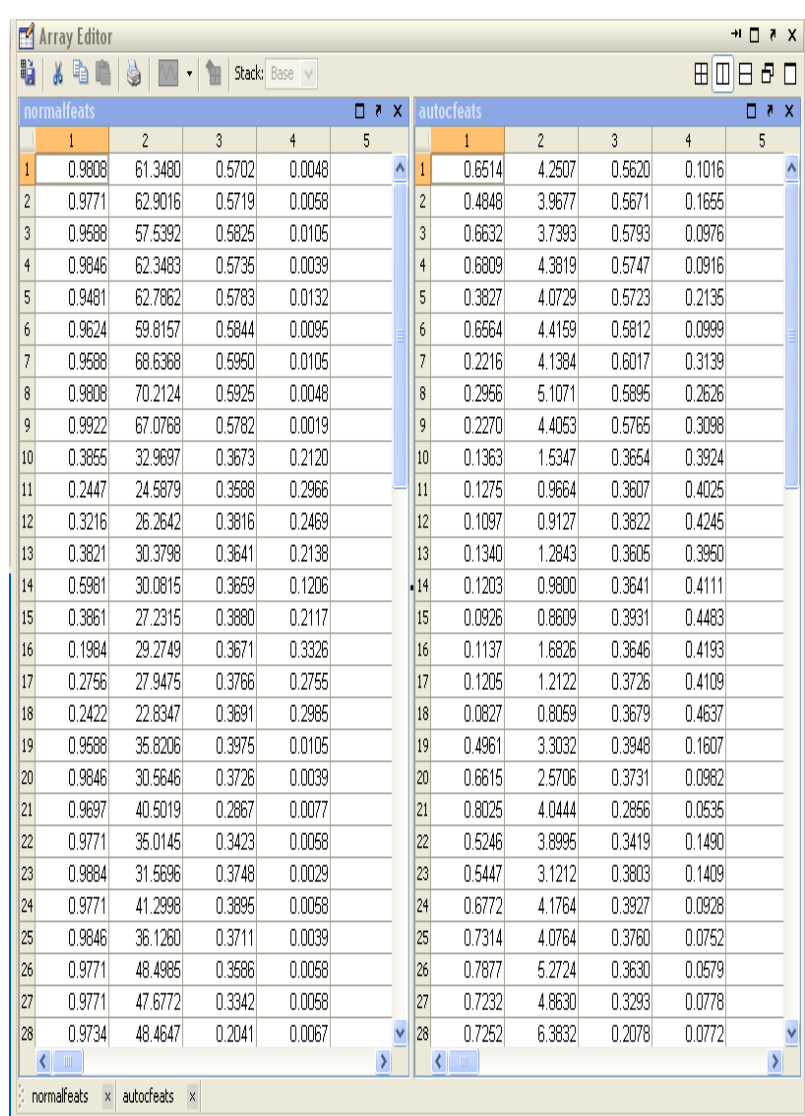

Figure 5: Feature set obtained during training phase for 72 subimages in the database

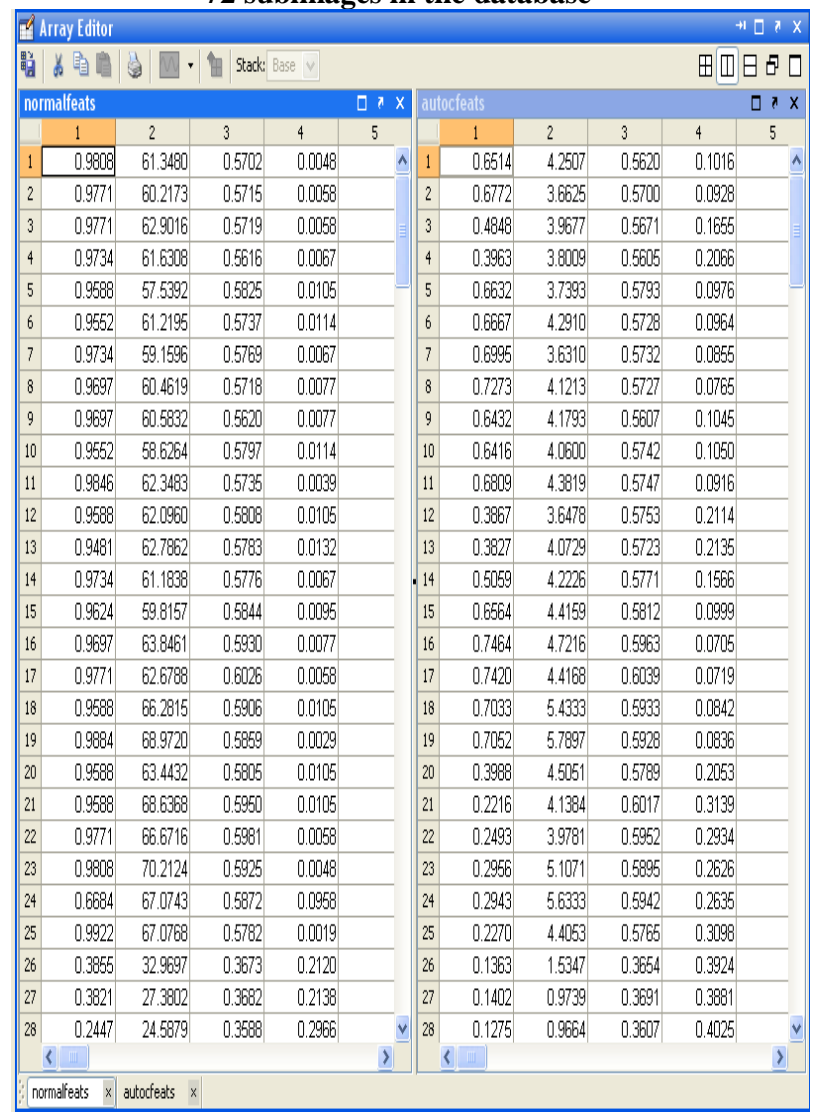

Figure 6: Feature set obtained during training phase for 200 subimages in the database

\section{CONCLUSION}

A new perceptual model based on a set of computational measures corresponding to perceptual textural features namely coarseness, directionality, contrast, and busyness is proposed. Computational measures are based on multiple representations: original image representation and the autocorrelation function associated with original images. Coarseness was estimated as an average of the number of maxima. Contrast was estimated as a combination of the average amplitude of the gradient, the percentage of pixels having the amplitude superior to a certain threshold and coarseness itself. Directionality was estimated as the average number of pixels having the dominant orientation(s). Busyness was estimated based on coarseness. These four basic properties of texture were conceptually defined. The conceptual expressions were put into computational forms. In this approach, autocorrelation function was computed for a given image, and the features were derived from these autocorrelated and original images. In order to validate the proposed set of computational feature measures, applied them in classification and retrieval task on brodatz images. In both applications, better results in terms of classification rate and retrieval rate were obtained by using these developed features based on our approach.

\section{FUTURE WORK}

The immediate prospect related to this work is consideration of other perceptual features such as regularity and complexity. Application of these additional computational feature measures with existing features for texture classification and retrieval on large set of images from other texture database.

\section{REFERENCES}

[1] R. M. Haralick and L. G. Shapiro, Computer and Robot Vision. Reading, MA: Addison-Wesley, 1992, vol. 1.

[2] R. Karu, A. K. Jain, and R. M. Bolle, "Is there any texture in the image?," Pattern Recognit., vol. 29, no. 9, pp. 1437-1466, 1996.

[3] J. Zhang and T. Tan, "Brief review of invariant texture analysis methods," Pattern Recognit., vol. 35, pp. 735 747, 2002.

[4] A.R. Rao, A Taxonomy for Texture Description and Identification. New York: Springer-Verlag, 1990.

[5] M. Galloway, "Texture analysis using gray-level run lengths," Computer Graphics Image Processing, vol. 4, pp. 172-199, 1974.

[6] Rosenfeld and M. Thursion, "Edge and curve detection for visual scene analysis," IEEE Trans. Comput., vol. C20, pp. 562-569, May 1971.

[7] J. S. Weszka, C. R. Dyer, and A. Rosenfeld, "A comparative study of texture measures for terrain classification," IEEE Trans. Syst. Man Cybern., vol. SMC-6, pp. 269-285, Apr 1976.

[8] P. C. Chen and T. Pavlidis, "Segmentation by texture using correlation," IEEE Trans. Pattern Anal. Machine Intell., vol. PAMI-5, pp. 64-69, 1983.

[9] B. H. McCornick and S. N. Jayamurthy, "Time series model for texture synthesis," J. Computing Inform. Sci., vol. 3, pp. 329-343, Dec 1983. 
[10] Epifanio.I, Ayala.G, "A random set view of texture classification”, IEEE Trans. Image Process., vol. 11, no. 8, pp. 859-867, Aug 2002.

[11] Zhi-Zong Wang, Jun-Hai Yong, "Texture Analysis and Classification with Linear Regression Model based on Wavelet Transform", IEEE Trans. Image Process., vol. 17 , no. 8, pp. 1421-1430, 2008.

[12] Khellah F.M," Texture Classification Using Dominant Neighborhood Structure," IEEE Trans. Image Process., vol. 20, no. 11, pp. 3270-3279, 2011.

[13] Li Liu, Fieguth.P, “ Texture Classification from Random Features", IEEE Trans. Pattern Anal. Machine Intell., vol.34, no.3, pp. 574-586, 2011.

[14] Xiuwen Liu, Deliang Wang , “ Texture Classification using Spectral Histograms", IEEE Trans. Image Process., vol. 12, no. 6, pp. 661-670, 2003.

[15] Campisi.p et.al , “ Robust Rotation- Invariant Texture Classification Using a Model Based Approach", IEEE Trans. Image Process., vol. 13, no. 6, pp. 782-791, 2004.

[16] R. M. Haralick, "Statistical and structural approaches to texture," Proc. IEEE, vol. 67, no. 5, pp. 786-804, May 1979.

[17] R. M. Haralick, K. Shanmugam, and I. Dinstein, "Textural features for image classification," IEEE Trans. Syst., Man Cybern., vol. 3, no. 6, pp. 610-621, Nov. 1973.

[18] R. Jain, R. Kasturi, and B. G. Schunck, Machine Vision. New York: McGraw-Hill, 1995.

[19] A.H. S. Solberg and A. K. Jain, "Texture analysis of SAR images: a comparative study," Norwegian Comput. Center and Michigan State Univ., Research Rep., 1997.

[20] M. Tuceryan and A. K. Jain, "Texture analysis," in Handbook of Pattern Recognition and Computer Vision, C. H. Chen, L. F. Pau, and P. S. P. Wang, Eds. Singapore: World Scientific, 1993.

[21] F. Tomita and S. Tsuji, Computer Analysis of Visual Textures. Norwell, MA: Kluwer, 1990.

[22] L. Van Gool, P. Dewaele, and A. Oosterlinck, "Texture analysis anno 1983," J. Comput. Vis. Graph. Image Process., vol. 29, pp. 336-357, 1985.

[23] B.Julesz, "Visual pattern discrimination," IRE Trans., Info. Theory, vol. IT-8, pp. 84092, Feb. 1962.

[24] B. Julesz, E. N Gilbert. L. A. Shepp, and H. L. Frisch. "Inability of humans to discriminate between visual textures that agree in secondorder statistics Revisited." Perception. vol. 2. pp. 391-405, 1973.
[25] B. Julesz, "Experiments in the visual perception of texture," Sci. Amer., vol. 232, no. 4, pp. 34-44, 1976.

[26] J. R. Bergen and E. H. Adelson, "Early vision and texture perception," Nature, vol. 333, no. 6171, pp. 363 364, May 1988

[27] Muwei Jian, Lei Liu, Cheng Yin, Lin Yuan, "Combining Perceptual Textural Features and Wavelet Features for Texture Classification”, pp. 238-241, May 2009.

[28] H. Tamura, S. Mori, and T. Yamawaki, "Textural features corresponding to visual perception," IEEE Trans. Syst., Man Cybern., vol. 8, no. 6, pp. 460-472, Jun. 1978

[29] M. Amadasun and R. King, "Textural features corresponding to textural properties," IEEE Trans. Syst., Man Cybern., vol. 19, no. 5, pp. 1264-1274, Sep.-Oct. 1989.

[30] R. Rao and G. L. Lohse, "Towards a texture naming system: Identifying relevant dimensions of texture," Vis. Res., vol. 36, no. 11, pp. 1649-1669, 1996.

[31] P. Brodatz, Textures: A Photographic Album for Artists and Designers. New York: Dover, 1966.

[32] Rosenfeld and E. B. Troy, "Visual texture analysis," Compt. Sci. Center, Univ. Maryland, College Park, Tech. Rep. TR-1 16, June 1970.

[33] K. C. Hayes, Jr., A. N. Shah, and A. Rosenfeld, "Texture coarseness: Further experiments," IEEE Trans. Syst. Man., Cybern., vol. SMC-4, pp. 467-472, Sept. 1974.

[34] R. Bajcsy, "Computer description of textured surfaces," in Proc. $3^{\text {rd }}$ Int. Joint Conf. Artificial Intelligence, Aug. 1973, pp. 572-579.

[35] Rosenfeld and A. C. Kak, Digital Picture Processing. New York: L_ Academic, 1976, Chap. 10.

[36] N. Abbadeni, "Computational perceptual features for texture representation and retrieval," IEEE Trans. Image Process., vol. 20, no. 1, pp. 236-246, Jan 2011.

[37] N. Abbadeni, D. Ziou, and S. Wang, "Computational measures corresponding to perceptual textural features," in Proc. 7th IEEE Int. Conf.Image Process., Vancouver, Canada, vol. 3, pp. 897-900, 2000.

[38] N. Abbadeni, D. Ziou, and S.Wang, "Autocovariancebased perceptual textural features corresponding to human visual perception," in Proc.15th IAPR/IEEE Int. Conf. Pattern Recognit., Barcelona, Spain, vol. 3, pp. 901-904, Sep. 3-8, 2000. 